type $\alpha_{2,4}$ is indecomposable; $\alpha_{2,8}$ has, apart from order, the unique decomposition into indecomposable factors:

$$
\alpha_{2,8}=\alpha_{1,2} \times \alpha_{2,4} ;
$$

and finally $\alpha_{2,12}$ has two different decompositions into indecomposable factors:

$$
\alpha_{2,12}=\alpha_{2,6} \times \alpha_{1,2}=\alpha_{2,4} \times \alpha_{1,3} .
$$

Thus the last example shows that the refinement theorems 4.7 and 4.8 , as well as the unique factorization theorem 4.9 , of Jónsson and Tarski cannot be extended to algebras which have an idempotent element but not a zero element. The problem whether the cancellation theorem 4.10 can be extended to such algebras still remains open.

University of California

\title{
NOTE ON A PAPER BY C. E. RICKART
}

R. P. DILWORTH AND MORGAN WARD

In a recent issue of this Bulletin, ${ }^{1}$ C. E. Rickart proves the following two theorems:

THEOREM 1. Any one-to-one multiplicative mapping of a Boolean ring onto an arbitrary ring is necessarily additive.

TheOREM 3. Any one-to-one meet preserving mapping of a distributive lattice onto a distributive lattice is also join preserving.

We should like to point out that both of these theorems are simple consequences of the following well known principle of lattice theory:

Any one-to-one mapping of one lattice onto another lattice which preserves order both ways is a lattice isomorphism.

Now a one-to-one meet preserving mapping of one lattice onto another preserves order both ways; for if $x$ and $x^{\prime}$ denote corresponding elements,

$$
a \geqq b \rightleftarrows a \cap b=b \rightleftarrows a^{\prime} \cap b^{\prime}=b^{\prime} \rightleftarrows a^{\prime} \geqq b^{\prime} .
$$

California Institute of Technology

Received by the editors September 20, 1948.

${ }^{1}$ Vol. 54 (1948) pp. 758-764. 